\title{
BEP/SEP and Outage Performance Analysis of $L$-Branch Maximal-Ratio Combiner for $\kappa-\mu$ Fading
}

\author{
Mirza Milišić, Mirza Hamza, and Mesud Hadžialić \\ Faculty of Electrical Engineering, University of Sarajevo, Zmaja od Bosne bb, 71000 Sarajevo, Bosnia And Herzegovina \\ Correspondence should be addressed to Mirza Milišić, mmilisic@etf.unsa.ba
}

Received 1 August 2008; Accepted 19 January 2009

Recommended by Chih-Yang Kao

Maximal-ratio combiner (MRC) performances in fading channels have been of interest for a long time, which can be seen by a number of papers concerning this topic. In this paper we treat bit error probability (BEP), symbol error probability (SEP) and outage probability of MRC in presence of $\kappa-\mu$ fading. We will present $\kappa-\mu$ fading model, probability density function (PDF), and cumulative distribution function (CDF). We will also present PDF, CDF, and outage probability of the $L$-branch MRC output. BEP/SEP will be evaluated for broad class of modulation types and for coherent and noncoherent types of detection. BEP/SEP and outage performances of the MRC will be evaluated for different number of branches via Monte Carlo simulations and theoretical expressions.

Copyright (C) 2009 Mirza Milišić et al. This is an open access article distributed under the Creative Commons Attribution License, which permits unrestricted use, distribution, and reproduction in any medium, provided the original work is properly cited.

\section{Introduction}

MRC performances in fading channels have been of interest for a long time, which can be seen by a numerous published papers concerning this topic. Most of these papers are concerned by Rayleigh, Nakagami-m, Hoyt (Nakagamiq), Rice (Nakagami- $n$ ), and Weibull fading [1-5]. Beside MRC, performances of selection combining, equal-gain combining, hybrid combining, and switched combining in fading channels have also been studied. Most of the papers treating diversity combining have examined only dual-branch combining because of the inability to obtain closed-form expressions for evaluated parameters of diversity system. Scenarios of correlated fading in combiner branches have also been examined in numerous papers. Nevertheless, depending on system used and combiner implementation, one must take care of resources available at the receiver, such as: space, frequency, and complexity. Moreover, fading statistic does not necessary have to be the same in each branch, for example, PDF can be the same, but with different parameters (Nakagami- $m$ fading in $i$ th and $j$ th branches, with $m_{i} \neq m_{j}$ ), or PDFs in different branches are different (Nakagami- $m$ fading in $i$ th branch, and Rice fading in $j$ th branch). This paper treats MRC outage performances in presence of $\kappa-\mu$ fading $[6,7]$. This type of fading has been chosen because it includes, as special cases, Nakagami$m$ and Nakagami- $n$ (Rice) fading, and their entire special cases as well (e.g., Rayleigh and one-sided Gaussian fading). It will be shown that the sum of $\kappa-\mu$ squares is $\kappa-\mu$ square as well (but with different parameters), which is an ideal choice for MRC analysis. Concerning this, in this paper, we will present model for $\kappa-\mu$ distribution and closed form expressions for outage probability, BEP and SEP at the MRC output will be derived for a broad class of modulation types. Based upon generic expressions for $\mathrm{BEP} / \mathrm{SEP}$ for coherent and noncoherent detection, BEP/SEP will be evaluated in further analysis. Outage and BEP/SEP performances will be presented for $L$-branch combining via Monte Carlo simulations and theoretical expressions. This paper is organized as follows. In Section 2, we review physical model of the distribution. In Section 3, we examine $\kappa-\mu$ MRC, and we show that the sum of $\kappa-\mu$ squares is $\kappa-\mu$ square. Throughout Section 4 we analyze BEP/SEP for $\kappa-\mu$ MRC based on generic expressions for BEP/SEP for coherent and noncoherent detection types for various modulation techniques. Discussion and simulation results are presented in Section 5, where some conclusions have been drawn. 


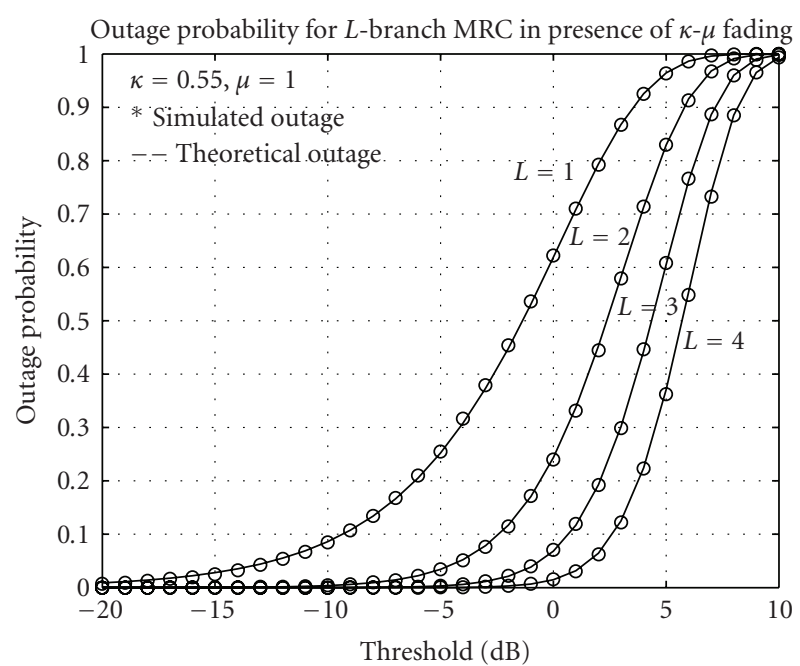

FIgURE 1: Outage probability for $\kappa=0.55$ and $\mu=1$.

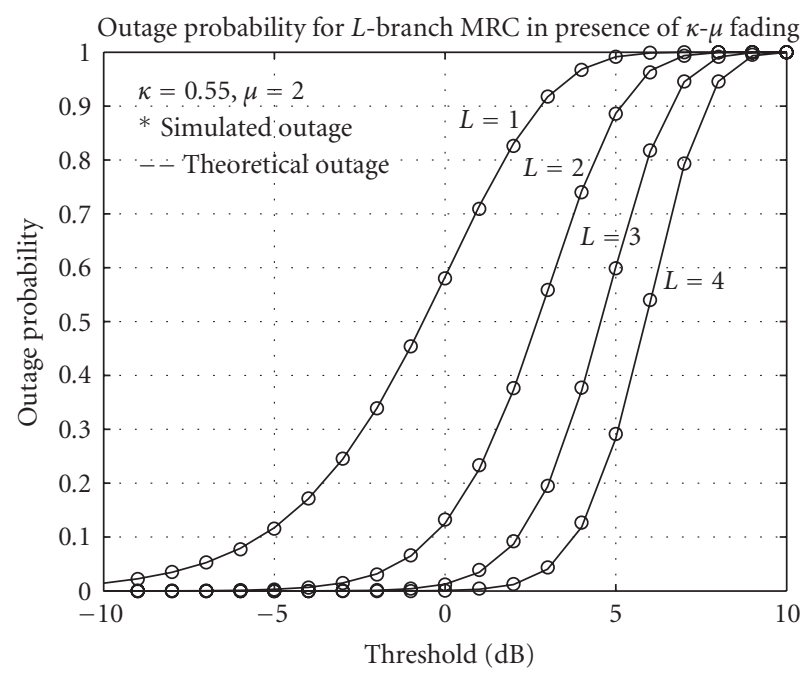

FIgURE 2: Outage probability for $\kappa=0.55$ and $\mu=2$.

\section{Physical Model of the $\kappa-\mu$ Distribution}

Physical model and derivation of the $\kappa-\mu$ distribution is described in [7]. Nevertheless, for the purpose of integrity of this paper and apprehension of generality of this model (as well as its applications to the MRC), it is necessary to revise the basics of the $\kappa-\mu$ distribution physical model. The fading model for the $\kappa-\mu$ distribution considers a signal composed of clusters of multipath waves, propagating in a nonhomogeneous environment. Within single cluster, the phases of the scattered waves are random and have similar delay times, with delay-time spreads of different clusters being relatively large. It is assumed that the clusters of multipath waves have scattered waves with identical powers, and that each cluster has a dominant component with arbitrary power. This distribution is well suited for lineof-sight (LoS) applications, since every cluster of multipath waves has a dominant component (with arbitrary power).
In special case, if we set all dominant components to zero, then this distribution can very well describe nonline-ofsight (NLoS) scenarios. Given the physical model for the $\kappa-\mu$ distribution, envelope $R$ and instantaneous power $\gamma$, can be written in terms of the inphase and quadrature components of the fading signal as

$$
R^{2}=\gamma=\sum_{i=1}^{n}\left(X_{i}+p_{i}\right)^{2}+\sum_{i=1}^{n}\left(Y_{i}+q_{i}\right)^{2},
$$

where $X_{i}$ and $Y_{i}$ are mutually independent Gaussian processes with $\overline{X_{i}}=\overline{Y_{i}}=0$ and $\overline{X_{i}^{2}}=\overline{Y_{i}^{2}}=\sigma^{2} \cdot p_{i}$ and $q_{i}$ are, respectively, the mean values of the inphase and quadrature components of the multipath waves of cluster $i$, and $n$ is the number of clusters of multipath.

By performing random variables (RVs) transformation, in accordance to [7, Section 2.2], we obtain the instantaneous power PDF of the $\kappa-\mu \mathrm{RV}$ :

$$
\begin{aligned}
f_{\gamma}(\gamma)= & \frac{1}{2 \sigma^{2}} \cdot\left(\frac{\gamma}{d^{2}}\right)^{(n-1) / 2} \\
& \times \exp \left(-\frac{\gamma+d^{2}}{2 \sigma^{2}}\right) \cdot I_{n-1}\left(\frac{d \sqrt{\gamma}}{\sigma^{2}}\right),
\end{aligned}
$$

where $d^{2}=\sum_{i=1}^{n} d_{i}^{2}$. It can be seen that

$$
\begin{gathered}
\Omega=\overline{R^{2}}=\bar{\gamma}=2 n \sigma^{2}+d^{2}, \\
\overline{R^{4}}=\overline{\gamma^{2}}=4 n \sigma^{4}+4 \sigma^{2} d^{2}+\left(2 n \sigma^{2}+d^{2}\right)^{2} .
\end{gathered}
$$

Therefore,

$$
\operatorname{Var}\left(R^{2}\right)=\operatorname{Var}(\gamma)=4 n \sigma^{4}+4 \sigma^{2} d^{2}
$$

Parameter $\kappa$ is defined as $\kappa=d^{2} / 2 n \sigma^{2}$ and represents the ratio between the total power of the dominant components and the total power of the scattered waves. Although $n$ can be expressed in terms of continuous physical parameters (mean-squared value of the power, the variance of the power, and $\kappa)$, it still has discrete nature. If these parameters are to be obtained by field measurements, the value of the parameter $n$ would be a real number (not an integer). Several reasons exist for this. One of them, and probably the most meaningful, is that although the model proposed here is general, it is in fact an approximate solution to the socalled random phase problem (which has been extensively elaborated in [7]), as are all the other well-known fading models approximate solutions to the random phase problem. The limitation of the model can be made less stringent by defining $\mu$ to be the real extension of $n$. Noninteger values of the parameter $\mu$ may account for: the non-Gaussian nature of the inphase and quadrature components of each cluster of the fading signal, nonzero correlation among the clusters of multipath components, nonzero correlation between inphase and quadrature components within each cluster, and so forth. Noninteger values of clusters have been found in practice, and are extensively reported in literature, for example, [8]. 


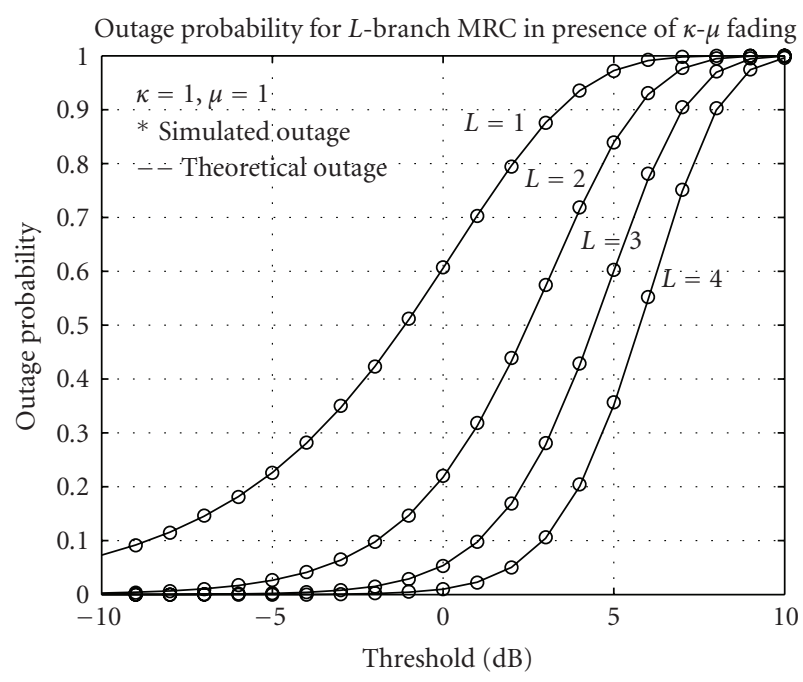

FIGURE 3: Outage probability for $\kappa=1$ and $\mu=1$.

Now, using the definitions for parameters $\kappa$ and $\mu$, and the considerations given above, the $\kappa-\mu$ power PDF can be written from (2) as

$$
\begin{aligned}
f_{\gamma}(\gamma)= & \frac{\mu(1+\kappa)^{(\mu+1) / 2}}{\kappa^{(\mu-1) / 2} \cdot \exp (\mu \kappa) \cdot \Omega^{(\mu+1) / 2}} \cdot \gamma^{(\mu-1) / 2} \\
& \times \exp \left[-\frac{\mu(1+\kappa) \gamma}{\Omega}\right] \cdot I_{\mu-1}\left[2 \mu \sqrt{\frac{\kappa(1+\kappa) \gamma}{\Omega}}\right] .
\end{aligned}
$$

From (5), $\kappa-\mu$ power CDF can be written in closed form as

$$
F_{\gamma}(\gamma)=\int_{0}^{\gamma} f_{\gamma}(x) d x=1-Q_{\mu}\left[\sqrt{2 \kappa \mu}, \sqrt{\frac{2(\kappa+1) \mu \gamma}{\Omega}}\right] \text {, }
$$

where

$$
Q_{\nu}(a, b)=\frac{1}{a^{\nu-1}} \int_{b}^{\infty} x^{\nu} \cdot \exp \left(-\frac{x^{2}+a^{2}}{2}\right) \cdot I_{\nu-1}(a x) \cdot d x
$$

is generalized Marcum $Q$ function [9], as stated in [7].

\section{3. $\kappa-\mu$ Maximal-Ratio Combiner}

There are four principal types of combining techniques [10] that depend essentially on the complexity restrictions put on the communication system and amount of channel state information (CSI) available at the receiver. As shown in [10], in the absence of interference, MRC is the optimal combining scheme, regardless of fading statistics, but most complex since MRC requires knowledge of all channel fading parameters (amplitudes, phases, and time delays). Since knowledge of channel fading amplitudes is needed for MRC, this scheme can be used in conjunction with unequal energy signals, such as M-QAM or any other

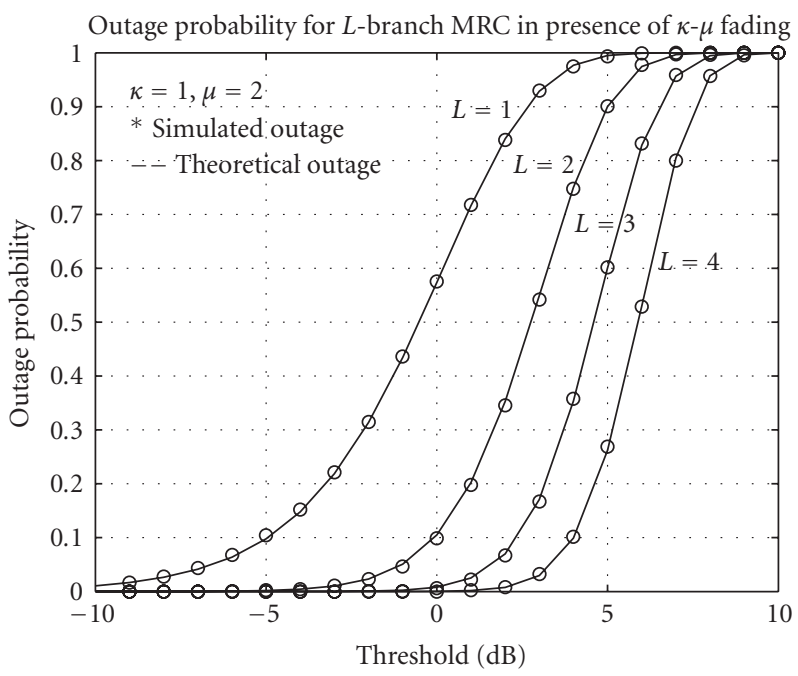

FIGURE 4: Outage probability for $\kappa=1$ and $\mu=2$.

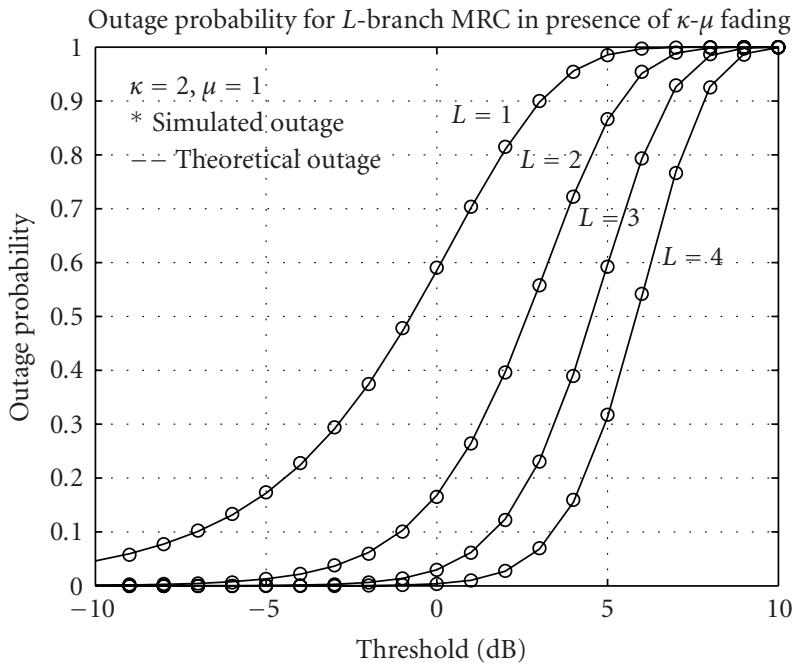

FIGURE 5: Outage probability for $\kappa=2$ and $\mu=1$.

amplitude/phase modulations. In this paper, we will treat $L$-branch MRC receiver. As shown in [10] MRC receiver is the optimal multichannel receiver, regardless of fading statistics in various diversity branches since it results in an ML receiver. For equally likely transmitted symbols, the total SNR per symbol at the output of the MRC is given by [11] $\gamma=\sum_{j=1}^{L} \gamma_{j}$, where $\gamma_{j}$ is instantaneous SNR in ith branch of $L$-branch MRC receiver. Repeating the same procedure as in Section, previous relation can be written in terms of inphase and quadrature components:

$$
\gamma=\sum_{j=1}^{L} \gamma_{i}=\sum_{j=1}^{L} R_{i}^{2}=\sum_{j=1}^{L} \sum_{i=1}^{n} R_{i, j}^{2},
$$

where $R_{i, j}^{2}$ represents total power of the $i$ th cluster manifested in $j$ th branch of the MRC receiver. Using (1) one can obtain

$$
\gamma=\sum_{j=1}^{L} \sum_{i=1}^{n}\left(X_{i, j}+p_{i, j}\right)^{2}+\left(Y_{i, j}+q_{i, j}\right)^{2} .
$$


Repeating the same procedure as in [7, Section 2.2] one can obtain Laplace transform of the PDF of the RV $\gamma$ (SNR):

$$
L\left\{f_{\gamma}(\gamma)\right\}=\prod_{j=1}^{L} L\left\{f_{\gamma_{j}}\left(\gamma_{j}\right)\right\}=\frac{\exp \left(-s d^{2} /\left(1+2 s \sigma^{2}\right)\right)}{\left(1+2 s \sigma^{2}\right)^{L \cdot n}},
$$

where $d^{2}=\sum_{j=1}^{L} d_{j}^{2}$. Inverse Laplace transform of (21) yields to PDF of the RV $\gamma$ :

$$
\begin{aligned}
f_{\gamma}(\gamma)= & \frac{L \mu(1+\kappa)^{(L \mu+1) / 2}}{\kappa^{(L \mu-1) / 2} \cdot \exp (L \mu \kappa) \cdot(L \Omega)^{(L \mu+1) / 2}} \cdot \gamma^{(L \mu-1) / 2} \\
& \times \exp \left[-\frac{\mu(1+\kappa) \gamma}{\Omega}\right] \cdot I_{\mu-1}\left[2 \mu \sqrt{L \frac{\kappa(1+\kappa) \gamma}{\Omega}}\right] .
\end{aligned}
$$

Note, that sum of $L$ squares of the $\kappa-\mu$ distributions is $\kappa-\mu$ distribution with different parameters, which means SNR at the output of the MRC receiver subdue to the $\kappa-\mu$ distribution with parameters

$$
\mu_{\mathrm{MRC}}=L \cdot \mu, \quad \kappa_{\mathrm{MRC}}=\kappa, \quad \Omega_{\mathrm{MRC}}=L \cdot \Omega .
$$

Now, it is easy to obtain CDF

$$
F_{\gamma}(\gamma)=\int_{0}^{\gamma} f_{\gamma}(x) d x=1-Q_{L \mu}\left[\sqrt{2 L \kappa \mu}, \sqrt{\frac{2(\kappa+1) \mu \gamma}{\Omega}}\right] .
$$

For fixed threshold, $\gamma_{\text {th }}$, outage probability is given by

$$
\begin{aligned}
P_{\text {out }}\left(\gamma_{\text {th }}\right) & =F_{\gamma}\left(\gamma_{\text {th }}\right)=\int_{0}^{\gamma_{\text {th }}} f_{\gamma}(x) d x \\
& =1-Q_{L \mu}\left[\sqrt{2 L \kappa \mu}, \sqrt{\frac{2(\kappa+1) \mu \gamma_{\text {th }}}{\Omega}}\right] .
\end{aligned}
$$

\section{SEP for $\kappa-\mu$ Maximal-Ratio Combiner}

When we analyze SEP, we must focus upon single modulation format because different modulations result in different SEPs. We must also consider type of detection (coherent or noncoherent). Although coherent detection results in smaller SEP than corresponding noncoherent detection for the same SNR, sometimes it is suitable to perform noncoherent detection depending on receiver structure complexity.

4.1. Noncoherent Detection. To obtain average SEP at MRC output for $\kappa-\mu$ fading for noncoherent detection, we will use generic expression for instantaneous SEP: SEP $=a \cdot \exp (-b \cdot$ $\gamma$ ), where $\gamma$ represents instantaneous SNR at MRC output for $\kappa-\mu$ fading, and nonnegative parameters $a$ and $b$ depend on used modulation format (see Table 1).

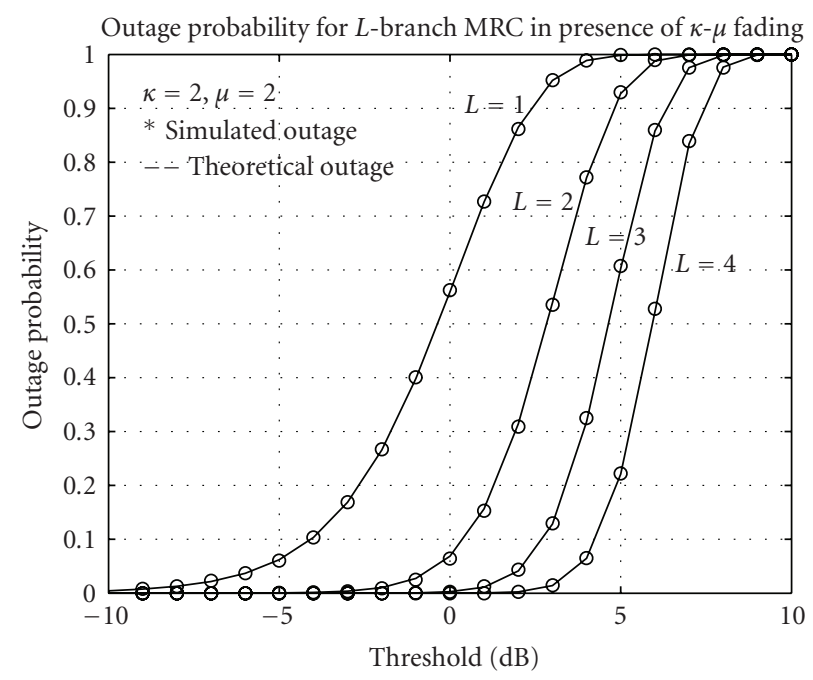

FIGURE 6: Outage probability for $\kappa=2$ and $\mu=2$.

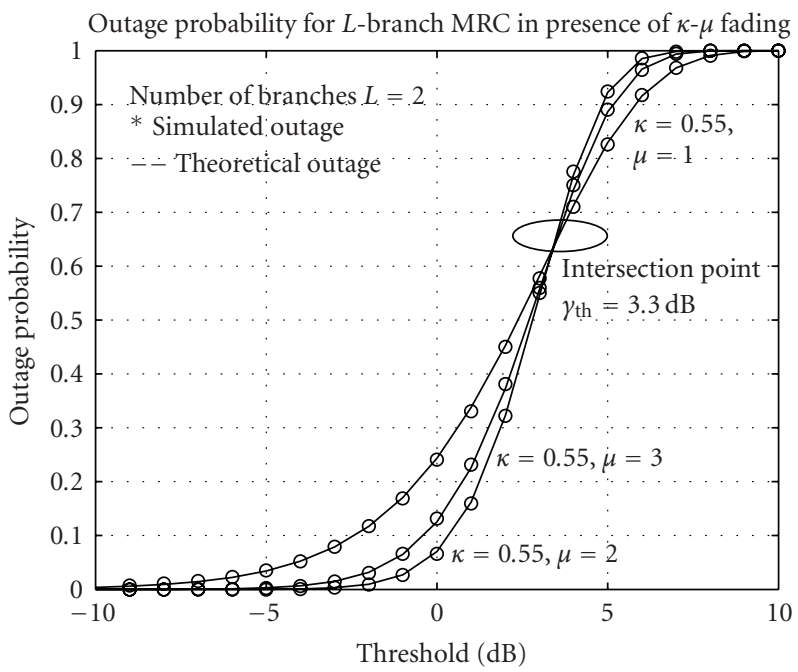

FIGURE 7: Outage probability for dual-branch MRC.

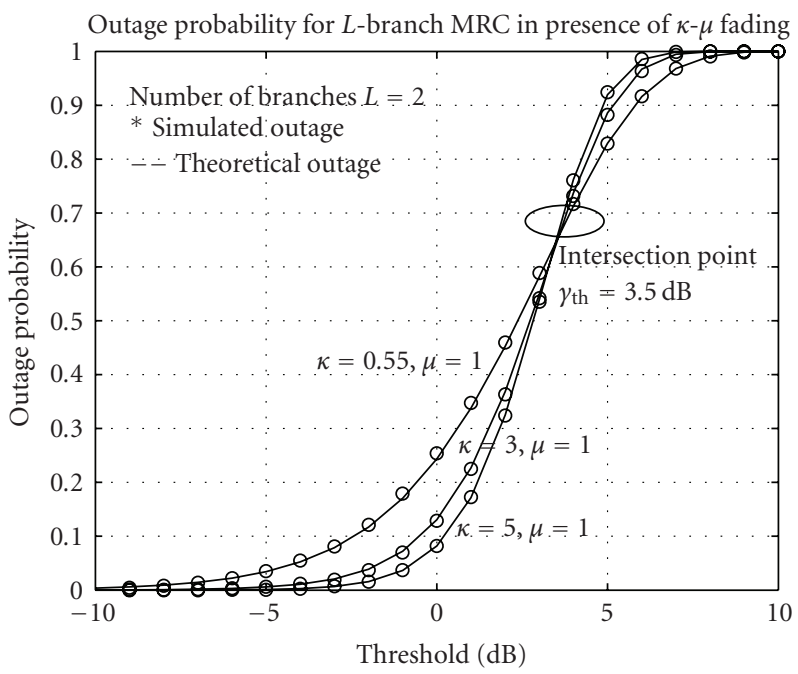

FIGURE 8: Outage probability for dual-branch MRC. 


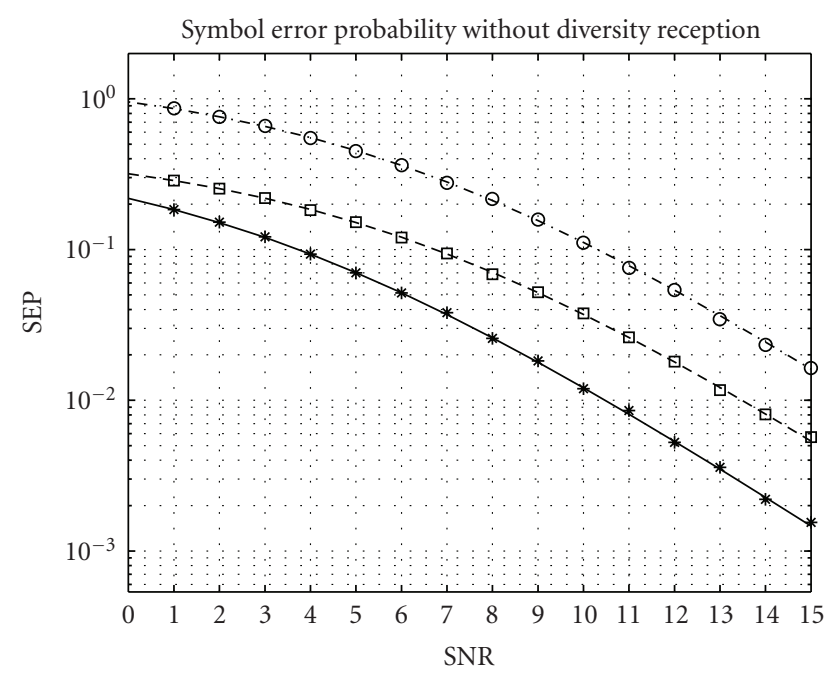

$\begin{aligned}-- & \text { NC-BFSK, } \kappa=0.55, \mu=2 \\ \text { - } & \text { NC-BPSK, } \kappa=0.55, \mu=2 \\ -- & \text { NC-MFSK, } M=4, \kappa=0.55, \mu=2 \\ \text { } & \text { NC-BFSK-sim, } \kappa=0.55, \mu=2 \\ * & \text { NC-BPSK-sim, } \kappa=0.55, \mu=2 \\ \circ & \text { NC-MFSK-sim, } M=4, \kappa=0.55, \mu=2\end{aligned}$

FIGURE 9: BEP/SEP for noncoherent detection, no diversity.

TABLE 1: Values of $a$ and $b$ for some noncoherent modulations.

\begin{tabular}{lccc}
\hline$a$ & & $b$ & \\
& 0.5 & & 1 \\
\hline 0.5 & BFSK & DBPSK \\
1 & - & - \\
$(M-1) / 2$ & MFSK & - \\
\hline
\end{tabular}

Average SEP can be obtained from

$$
\begin{aligned}
\overline{\mathrm{SEP}}= & \int_{0}^{+\infty} a \cdot \exp (-b \cdot \gamma) \cdot f_{\gamma}(\gamma) \cdot d \gamma \\
= & \int_{0}^{+\infty} a \cdot \frac{L \mu(1+\kappa)^{(L \mu+1) / 2}}{\kappa^{(L \mu-1) / 2} \cdot \exp (L \mu \kappa) \cdot(L \Omega)^{(L \mu+1) / 2}} \cdot \gamma^{(L \mu-1) / 2} \\
& \times \exp \left[-\gamma\left(b+\frac{\mu(1+\kappa)}{\Omega}\right)\right] \\
& \cdot I_{L \mu-1}\left[2 \mu \sqrt{L \frac{\kappa(1+\kappa) \gamma}{\Omega}}\right] \cdot d \gamma .
\end{aligned}
$$

Using [9, equation (5), page 318] we obtain closed-form expression for average SEP for noncoherent detection:

$$
\overline{\mathrm{SEP}}=a \cdot\left[\frac{\mu(1+\kappa)}{b \Omega+\mu(1+\kappa)} \cdot \exp \left(\frac{-b \kappa \Omega}{b \Omega+\mu(1+\kappa)}\right)\right]^{L \mu} .
$$

4.2. Coherent Detection. To obtain average SEP at MRC output for $\kappa-\mu$ fading for coherent detection, we will use
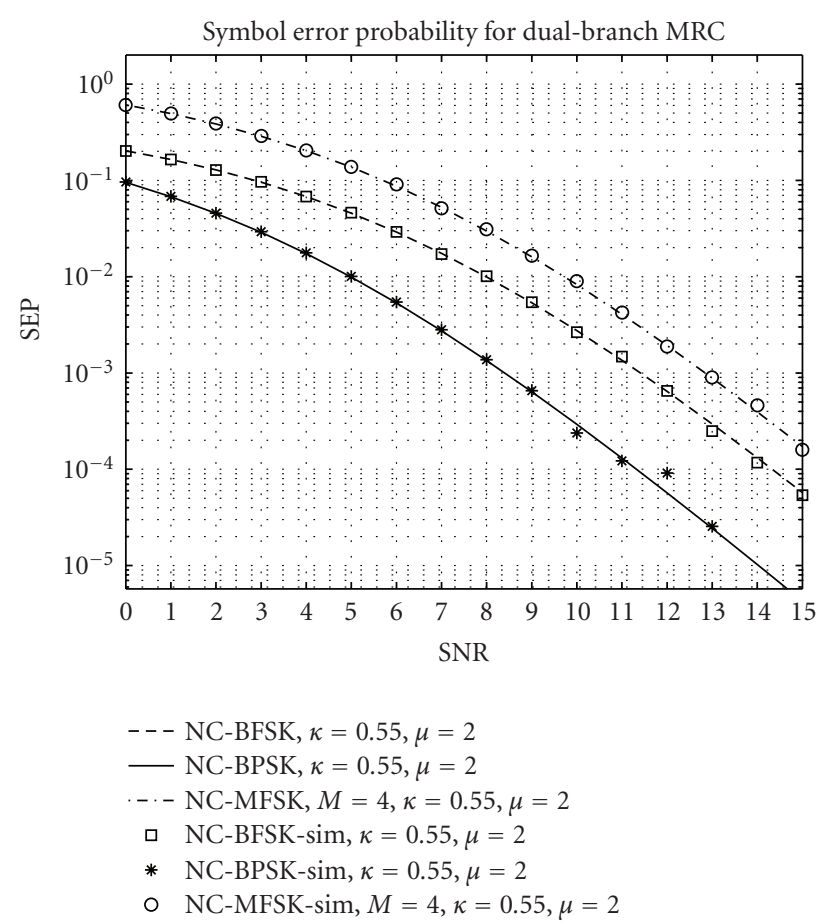

FIGURE 10: BEP/SEP for noncoherent detection, dual-branch diversity.

TABLE 2: Values of $a$ and $b$ for some coherent modulations.

\begin{tabular}{lcccc}
\hline$a$ & \multicolumn{4}{c}{$b$} \\
& 1 & 2 & $2 \sin ^{2}(\pi / M)$ & $3 /(M-1)$ \\
\hline 1 & BFSK & BPSK & - & - \\
2 & QPSK & DBPSK & MPSK & - \\
$4((\sqrt{M}-1) / \sqrt{M})$ & - & - & - & Rect. QAM \\
\hline
\end{tabular}

generic expression for instantaneous SEP: SEP $=a$. $Q(\sqrt{b \cdot \gamma})$, where $\gamma$ represents instantaneous SNR at MRC output for $\kappa-\mu$ fading, $Q(\cdot)$ function is defined as

$$
Q(x)=\frac{1}{\sqrt{2 \pi}} \int_{x}^{+\infty} \exp \left(\frac{-t^{2}}{2}\right) \cdot d t
$$

and nonnegative parameters $a$ and $b$ depend on used modulation format (see Table 2).

Average SEP can be obtained from

$$
\overline{\mathrm{SEP}}=\int_{0}^{+\infty} a \cdot Q(\sqrt{b \cdot \gamma}) \cdot f_{\gamma}(\gamma) \cdot d \gamma .
$$

Nevertheless, it is impossible to find closed-form solution for (18). Because of that we have to find adequate approximation of the $Q$ function. Knowing the continued fraction representation of the $Q$ function [12, equation (06.27.10.0001.01)], and adopting the first-order approximation:

$$
Q(x) \approx \frac{1}{x \sqrt{2 \pi}} \cdot \exp \left(\frac{-x^{2}}{2}\right)
$$




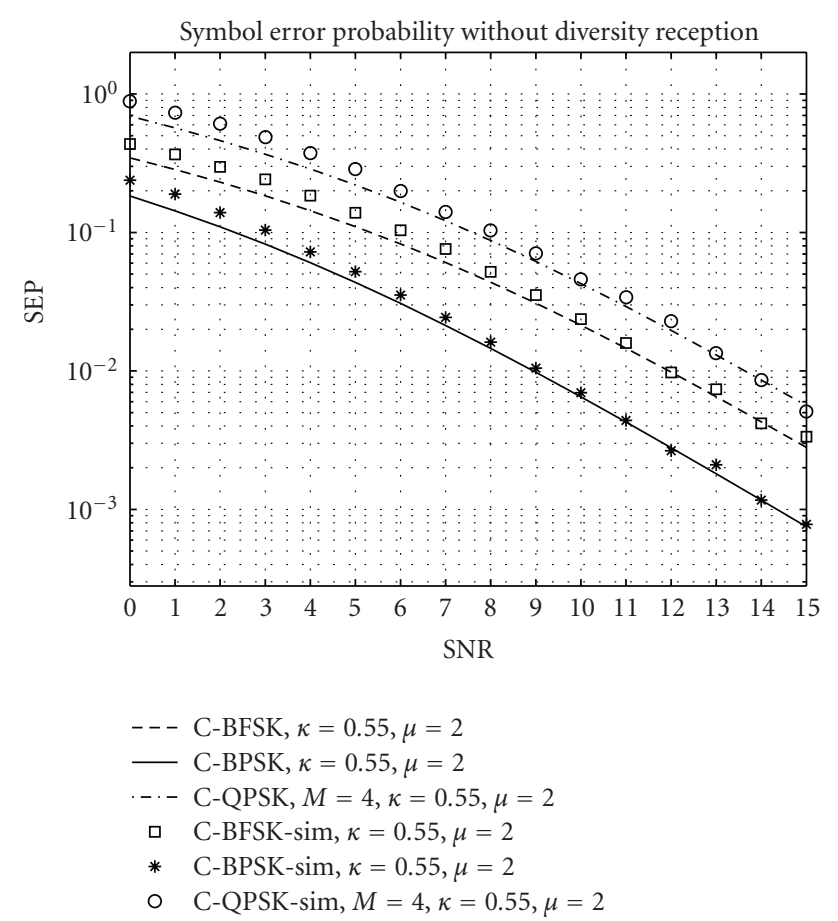

FIgURE 11: BEP/SEP for coherent detection, no diversity.

equation (18) now becomes

$$
\begin{aligned}
\overline{\mathrm{SEP}} \approx & \int_{0}^{+\infty} \frac{a}{\sqrt{2 \pi b \cdot \gamma}} \cdot \exp \left(\frac{-b \cdot \gamma}{2}\right) \cdot f_{\gamma}(\gamma) \cdot d \gamma \\
= & \int_{0}^{+\infty} \frac{a}{\sqrt{2 \pi b}} \cdot \frac{L \mu(1+\kappa)^{(L \mu+1) / 2}}{\kappa^{(L \mu-1) / 2} \cdot \exp (L \mu \kappa) \cdot(L \Omega)^{(L \mu+1) / 2}} \cdot \gamma^{(L \mu-2) / 2} \\
& \times \exp \left[-\gamma\left(\frac{b}{2}+\frac{\mu(1+\kappa)}{\Omega}\right)\right] \\
& \cdot I_{L \mu-1}\left[2 \mu \sqrt{L \frac{\kappa(1+\kappa) \gamma}{\Omega}}\right] \cdot d \gamma .
\end{aligned}
$$

Using [9, equation (5), page 318] we obtain closed-form expression for average SEP for coherent detection:

$$
\begin{aligned}
\overline{\mathrm{SEP}} \approx & \frac{a \cdot \Gamma(L \mu-0.5)}{\sqrt{2 \pi b} \cdot \Gamma(L \mu)} \cdot\left(\frac{\mu(1+\kappa)}{\exp (\kappa) \Omega}\right)^{L \mu} \\
& \times\left(\frac{b}{2}+\frac{\mu(1+\kappa)}{\Omega}\right)^{0.5-L \mu} \\
& \times{ }_{1} F_{1}\left(L \mu-0.5 ; L \mu ; \frac{\mu^{2} L \kappa(1+\kappa)}{b \Omega / 2+\mu(1+\kappa)}\right),
\end{aligned}
$$

where ${ }_{1} F_{1}(\cdot ; \cdot ; \cdot)$ is the Kummer confluent hypergeometric function defined in [12, equation (07.20.02.0001.01)].

\section{Simulations and Discussion of the Results}

As mentioned previously, MRC outage performances will be examined via Monte Carlo simulations and theoretical

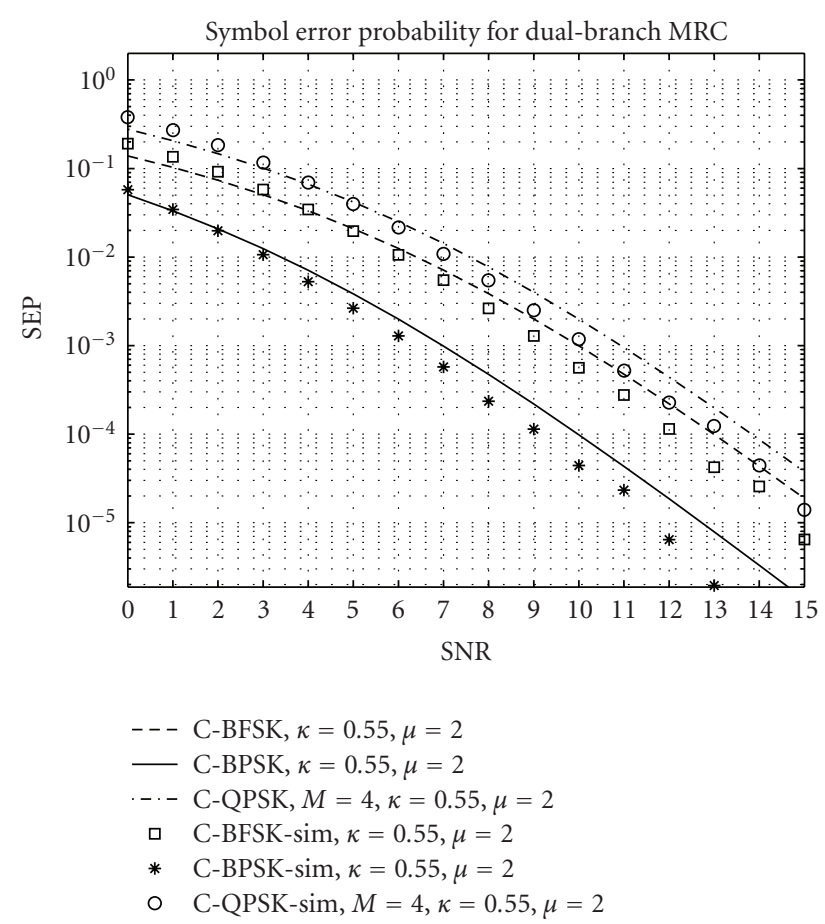

FIGURE 12: BEP/SEP for coherent detection, dual-branch diversity.

expressions (14). Figures 1, 2, 3, 4, 5, 6, 7, and 8 show theoretical and simulated outage probabilities as functions of threshold level $\gamma_{\text {th }} \cdot \gamma_{\text {th }}$ ranges from $-10 \mathrm{~dB}$ to $10 \mathrm{~dB}$. Figures 1-8 clearly show that theoretical expressions used are correct because theoretical results concur with simulations results extremely well. Figures $1-6$ show outage probability for $L=$ $1,2,3,4, \kappa=0.55,1,2$ and $\mu=1,2$. For fixed values of $\kappa$ and $\mu$ outage probabilities have been compared for specified numbers of combiners branches, $L$.

From Figures 1-6 it can be easily concluded that for fixed values of $\kappa$ and $\mu$ there is not much sense in increasing the number of branches (in many cases it is not economically or technically justified). We can also observe that the highest gain is obtained between curves for $L=1$ and $L=2$ (situation with no combining and dual-branch combining). Distribution parameters also have a significant impact on outage probability. When $\kappa$ is increasing, $P_{\text {out }}$ is decreasing. Namely, these results were expected because $\kappa$ represents ratio between total power of dominant components and total power of scattered components. Parameter $\mu$ represents fading severity parameter. As $\mu$ decreases, fading severity increases and so does outage probability. From Figures 16 , for fixed $\kappa$, as $\mu$ increases so does the slope of the outage curve. For dual-branch combining $(L=2)$, behavior of $P_{\text {out}}$, for different values of parameters $\kappa$ and $\mu$, can be observed in Figures 7 and 8. In Figure 7 parameter $\kappa$ is fixed, and parameter $\mu$ changes, and in Figure 8 we have inverse situation ( $\mu$ is fixed, and $\kappa$ changes). We perceive existence of the single intersection point (point where all curves intersect), and it is determined with only one parameter $(\kappa$ or $\mu$ ) and fixed number of branches $L$. In that point, outage probability $P_{\text {out }}$, and threshold level $\gamma_{\text {th }}$, are the same for 

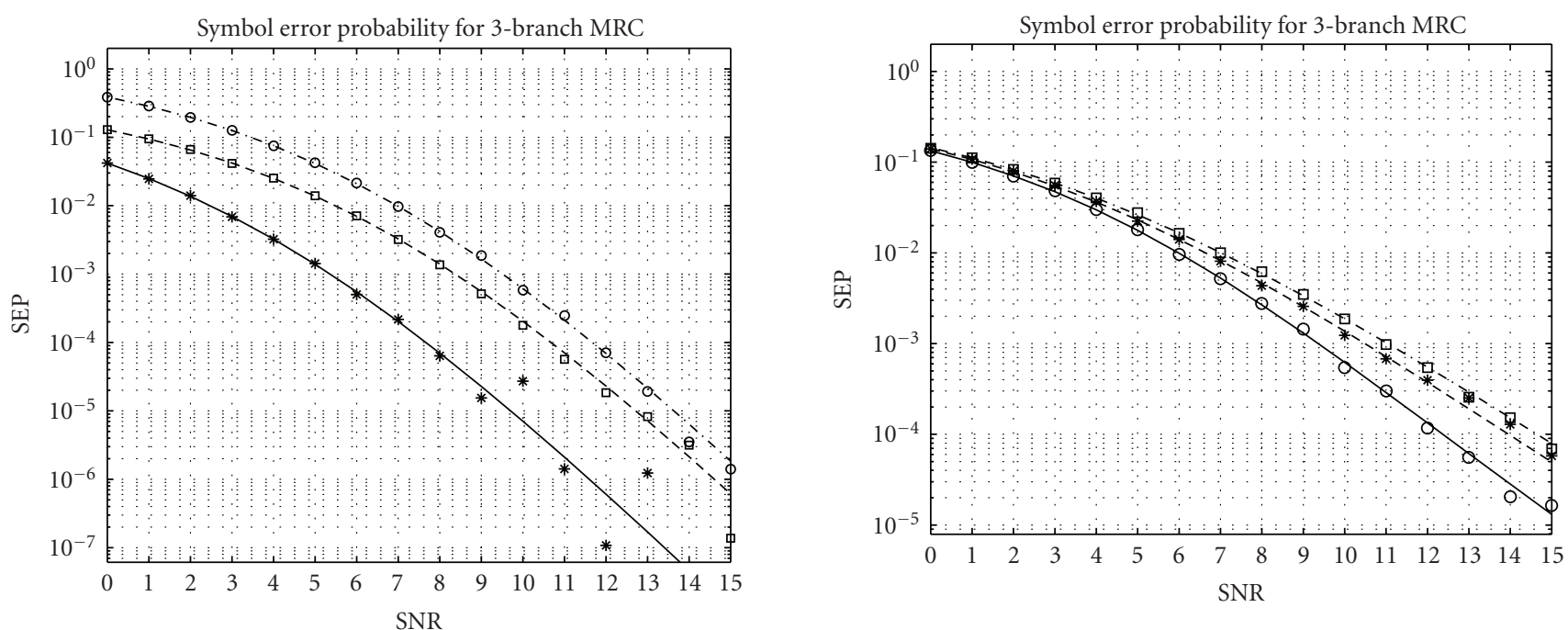

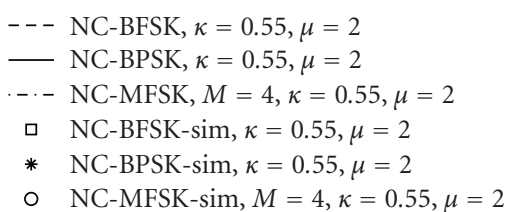

FIGURE 13: BEP/SEP for noncoherent detection, 3-branch diversity.

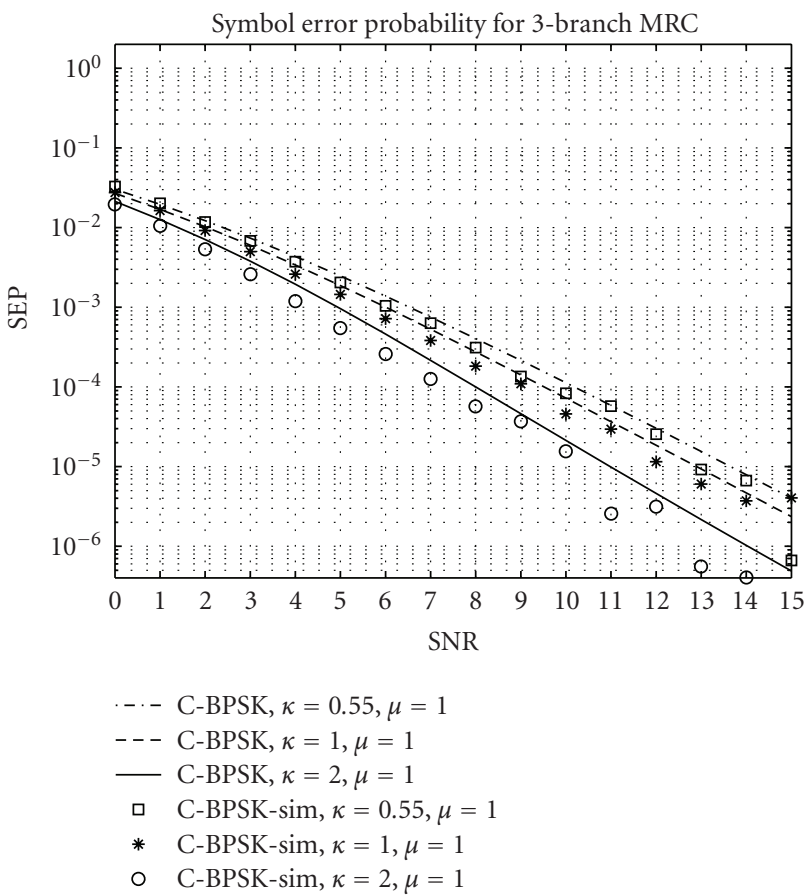

FIGURE 14: BEP/SEP for coherent detection, 3-branch diversity.

all curves (Figures 7 and 8). This point is also an inflexion point. If the threshold value is below the threshold value at inflexion point, channel dynamic is dominant, and if the threshold value is above the threshold value at inflexion point, receiver sensitivity is dominant. Namely, for smaller $\kappa$ and $\mu$, dynamic in channel is larger. If the threshold is set high

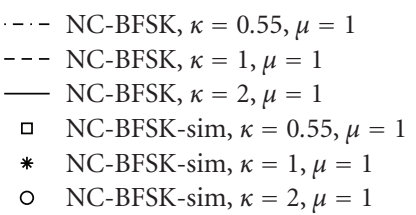

FIGURE 15: BEP/SEP for noncoherent detection, 3-branch diversity.

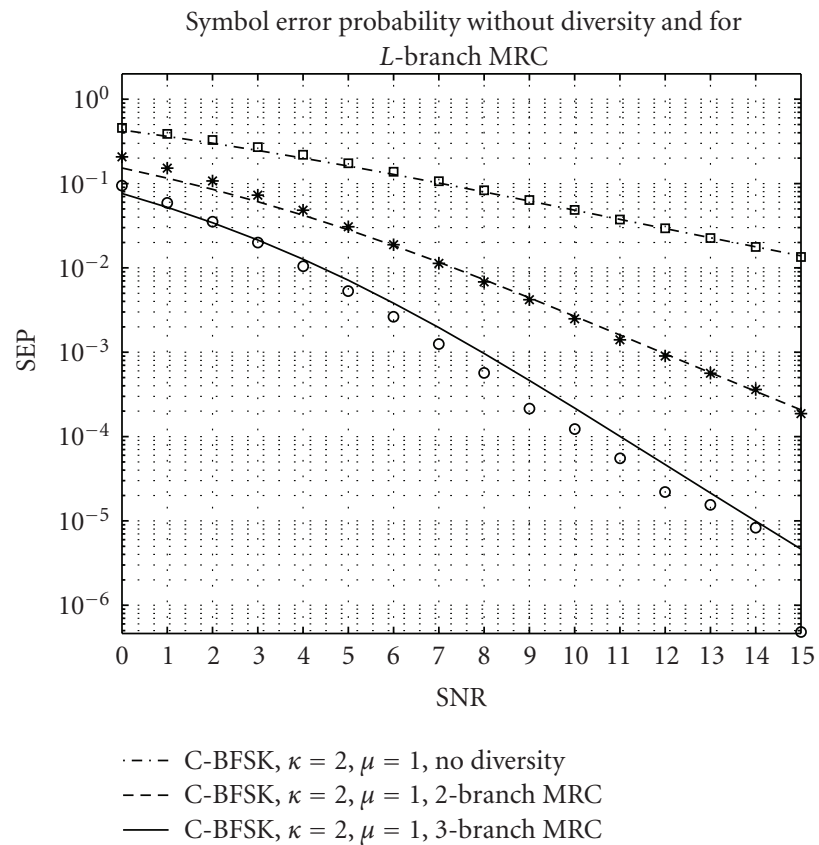

FIGURE 16: BEP/SEP for coherent detection, $L=1,2,3$.

enough, then it is logical to have smaller outage probability with larger channel dynamic apart from the case of smaller channel dynamic. MRC BEP/SEP, for both coherent and noncoherent detection, will be examined via Monte Carlo simulations and theoretical expressions (16) and (21) as well. In Figures 9-12 case of dual-branch combining has been shown because the highest gain is obtained between outage curves for $L=1$ and $L=2$ (situation with no combining 


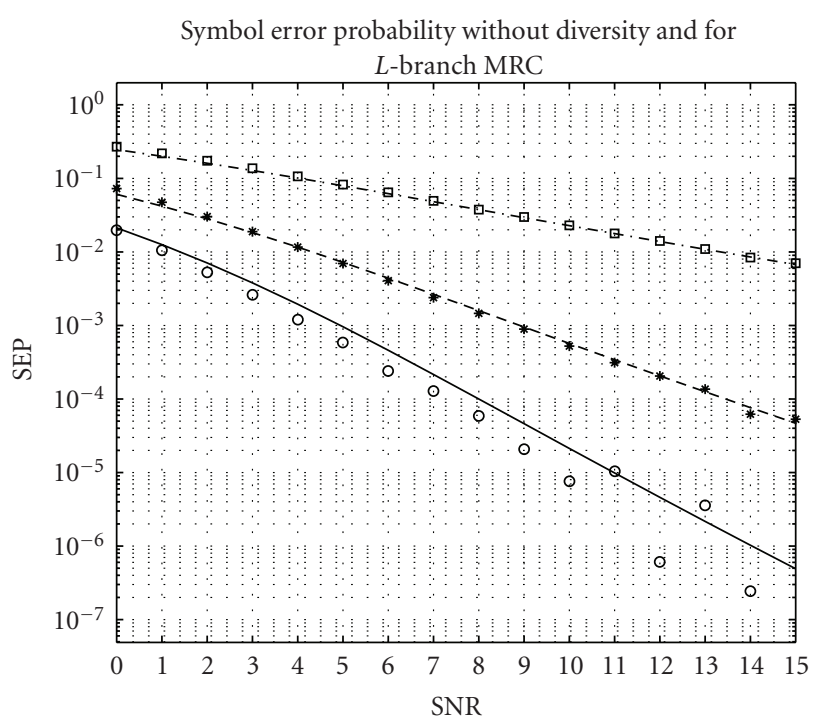

-. C-BPSK, $\kappa=2, \mu=1$, no diversity

-- - C-BPSK, $\kappa=2, \mu=1$, 2-branch MRC

- C-BPSK, $\kappa=2, \mu=1$, 3-branch MRC

FIgURE 17: BEP/SEP for coherent detection, $L=1,2,3$.

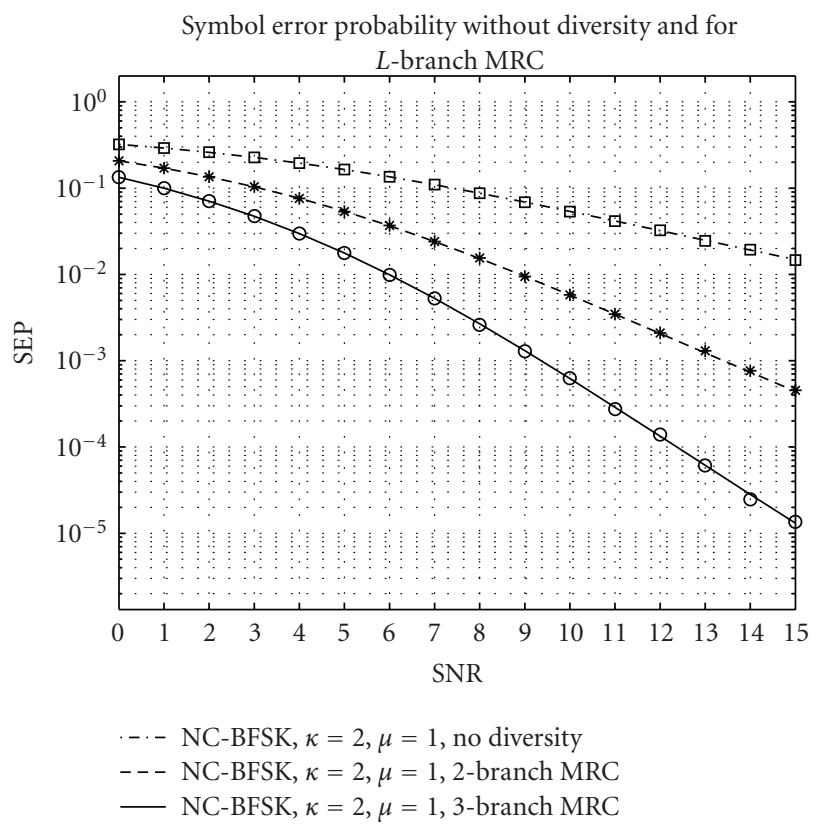

FIgURE 18: BEP/SEP for noncoherent detection, $L=1,2,3$.

and dual-branch combining). Figures 9-12 show theoretical and simulated average BEP/SEP as functions of average SNR $\Omega$. $\Omega$ ranges from $0 \mathrm{~dB}$ to $15 \mathrm{~dB}$. Figures $9-12$ clearly show that theoretical expressions used are correct because theoretical results concur with simulations results extremely well, but certain deviations of theory from simulation are noticeable in Figures 11 and 12 for a low values of $\Omega$. This is a consequence of the approximation used for generic expression for coherent detection (19). Figures 9 and 10 show BEP/SEP for $L=1$ and $L=2$, respectively, for noncoherent detection, and Figures 11 and 12 show BEP/SEP for $L=1$ and $L=2$, respectively, for coherent detection. By examining Figures 9 and 10 we notice that if we use dual-branch MRC we will gain $4 \mathrm{~dB}$ for the same BEP/SEP. The same goes for Figures 11 and 12, but we will gain approximately $7 \mathrm{~dB}$, which is to be expected because there is approximately $3 \mathrm{~dB}$ gain when we use coherent detection instead of noncoherent.

Figures 13, 14, and 15 show comparison between FSK and PSK for 3-branch combining. For Figures 13-15 various values of $\kappa$ and $\mu$ have been used, for both coherent and noncoherent detection. As we can observe, theoretical and simulation results concur very well. We can also observe gain obtained between no combining, dual-branch combining, and 3-branch combining cases in Figures 16, 17, and 18. As number of branches increases, BEP/SEP decreases, as expected.

\section{References}

[1] S. W. Kim, Y. G. Kim, and M. K. Simon, "Generalized selection combining based on the log-likelihood ratio," in Proceedings of IEEE International Conference on Communications (ICC '03), vol. 4, pp. 2789-2794, Anchorage, Alaska, USA, May 2003.

[2] A. Annamalai and C. Tellambura, "Analysis of hybrid selection/maximal-ratio diversity combiners with Gaussian errors," IEEE Transactions on Wireless Communications, vol. 1, no. 3, pp. 498-511, 2002.

[3] D. B. da Costa, M. D. Yacoub, and G. Fraidenraich, "Secondorder statistics for diversity-combining of non-identical, unbalanced, correlated Weibull signals," in Proceedings of the SBMO/IEEE MTT-S International Microwave and Optoelectronics Conference (IMOC '05), pp. 501-505, Brasilia, Brazil, July 2005.

[4] G. Fraidenraich, M. D. Yacoub, and J. C. S. Santos Filho, "Second-order statistics of maximal-ratio and equal-gain combining in Weibull fading," IEEE Communications Letters, vol. 9, no. 6, pp. 499-501, 2005.

[5] G. Fraidenraich, J. C. S. Santos Filho, and M. D. Yacoub, "Second-order statistics of maximal-ratio and equal-gain combining in Hoyt fading," IEEE Communications Letters, vol. 9, no. 1, pp. 19-21, 2005.

[6] J. C. S. Santos Filho and M. D. Yacoub, "Highly accurate $\kappa-\mu$ approximation to sum of $\mathrm{M}$ independent non-identical Ricean variates," Electronics Letters, vol. 41, no. 6, pp. 338-339, 2005.

[7] M. D. Yacoub, "The $\kappa-\mu$ distribution and the $\eta-\mu$ distribution," IEEE Antennas and Propagation Magazine, vol. 49, no. 1, pp. 68-81, 2007.

[8] H. Asplund, A. F. Molisch, M. Steinbauer, and N. B. Mehta, "Clustering of scatterers in mobile radio channels-evaluation and modeling in the COST259 directional channel model," in Proceedings of IEEE International Conference on Communications (ICC'02), vol. 2, pp. 901-905, New York, NY, USA, AprilMay 2002.

[9] A. P. Prudnikov, Yu. A. Brychkov, and O. I. Marichev, Integrals and Series: Direct Laplace Transforms, Gordon and Breach Science, Amsterdam, The Netherlands, 1992.

[10] M. K. Simon and M.-S. Alouini, Digital Communications over Fading Channels, John Wiley \& Sons, New York, NY, USA, 2nd edition, 2005.

[11] G. L. Stuber, Principles of Mobile Communications, Kluwer Academic Publishers, Norwell, Mass, USA, 1996.

[12] The Wolfram functions site, http://functions.wolfram.com. 

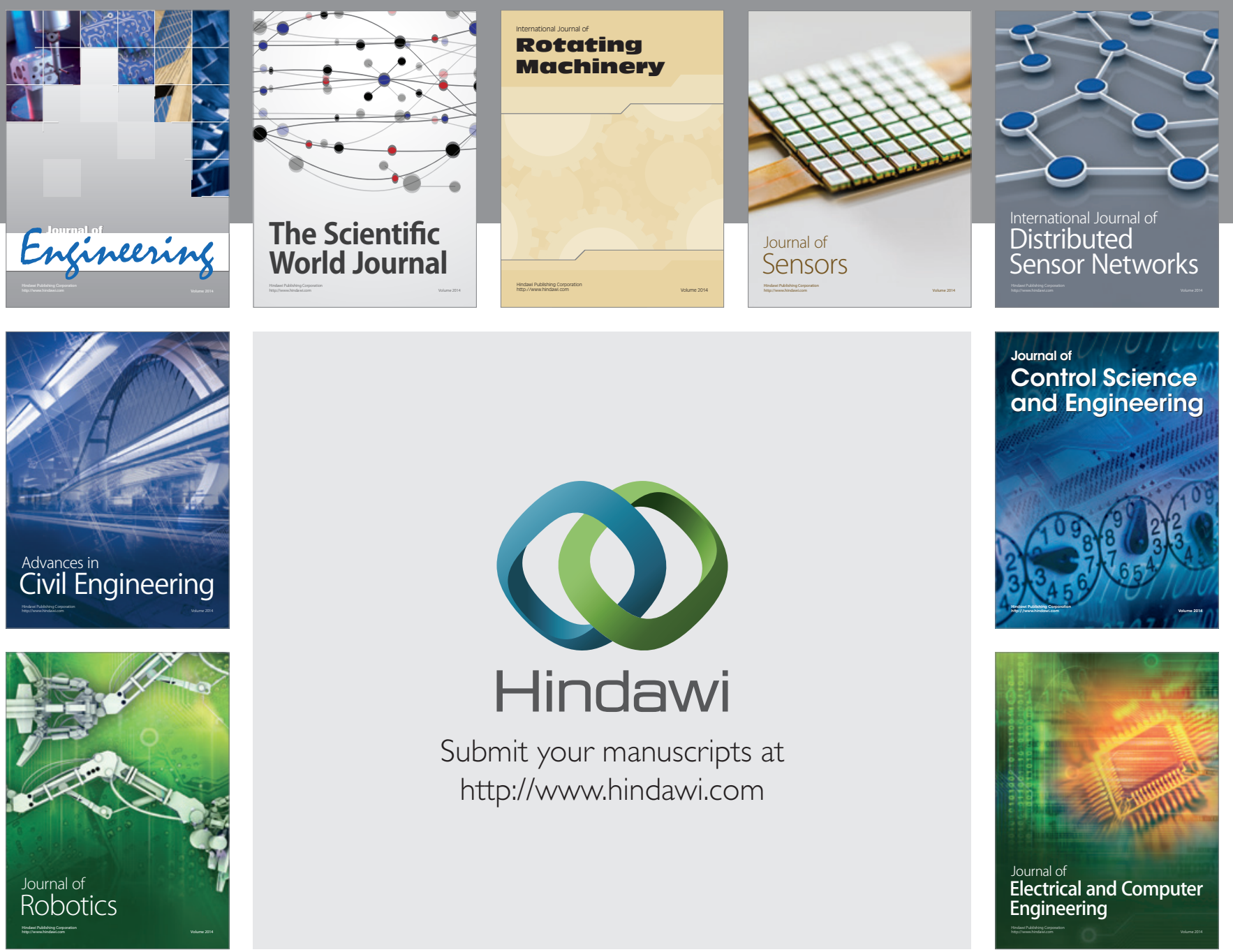

Submit your manuscripts at

http://www.hindawi.com
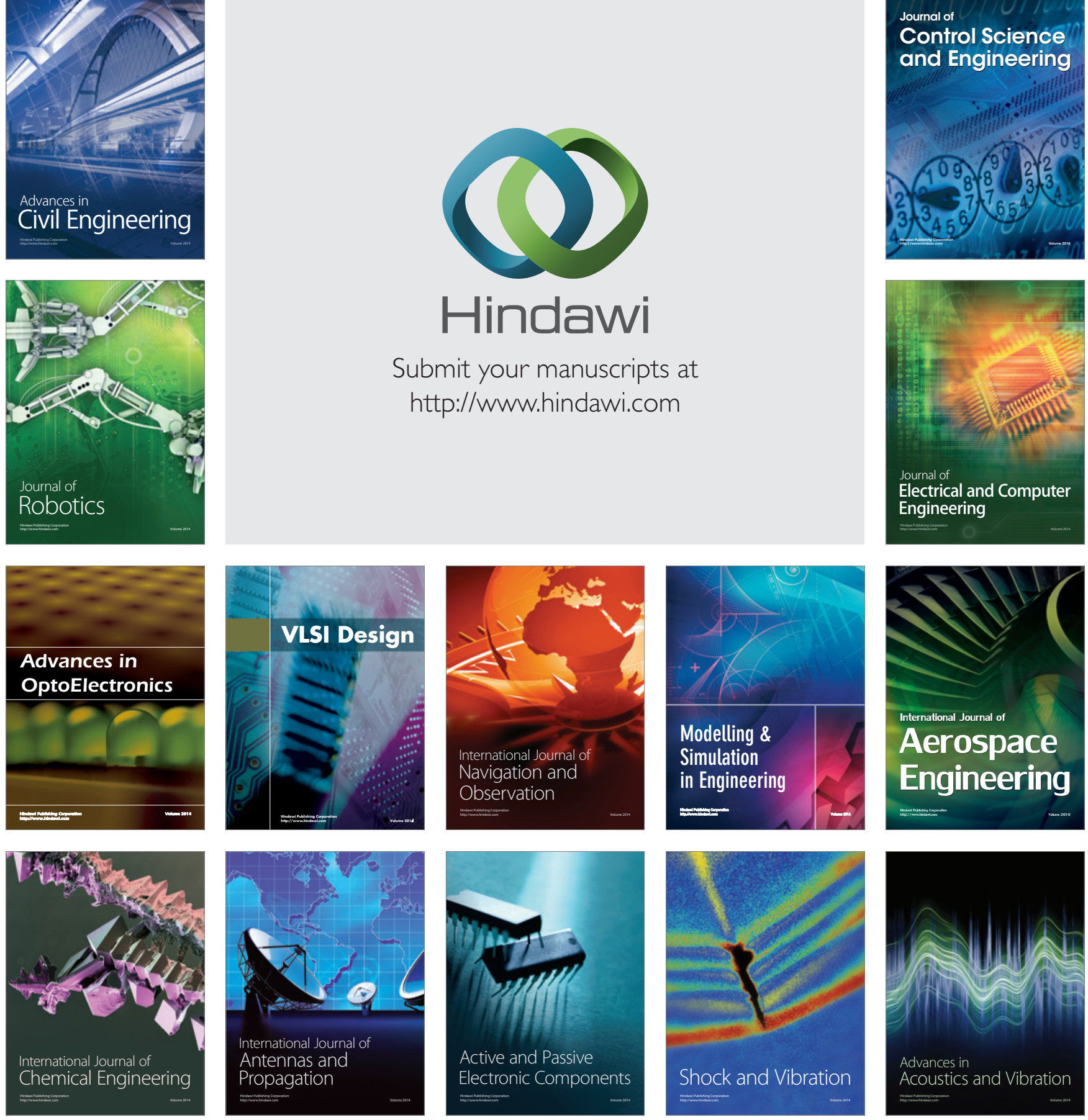